\title{
Study Protocol: A randomized controlled trial evaluating the effect of family-based behavioral treatment of childhood and adolescent obesity-The FABO-study
}

Hanna F. Skjåkødegård ${ }^{1, *^{*}}$, Yngvild S. Danielsen², Mette Morken ${ }^{1}$, Sara-Rebekka F. Linde ${ }^{1}$, Rachel P. Kolko ${ }^{3}$, Katherine N. Balantekin ${ }^{4,5,6,7}$, Denise E. Wilfley ${ }^{4,5,6,7}$ and Pétur B. Júlíusson ${ }^{1,8}$

\begin{abstract}
Background: The purpose of the FABO-study is to evaluate the effect of family-based behavioral social facilitation treatment (FBSFT), designed to target children's family and social support networks to enhance weight loss outcomes, compared to the standard treatment (treatment as usual, TAU) given to children and adolescents with obesity in a routine clinical practice.

Methods: Randomized controlled trial $(R C T)$, in which families $(n=120)$ are recruited from the children and adolescents (ages 6-18 years) referred to the Obesity Outpatient Clinic (OOC), Haukeland University Hospital, Norway. Criteria for admission to the $\mathrm{OOC}$ are BMI above the International Obesity Task Force (IOTF) cut-off $\geq 35$, or IOTF $\geq 30$ with obesity related co-morbidity. Families are randomized to receive FBSFT immediately or following one year of TAU. All participants receive a multidisciplinary assessment. For TAU this assessment results in a plan and a contract for chancing specific lifestyle behaviors. Thereafter each family participates in monthly counselling sessions with their primary health care nurse to work on implementing these goals, including measuring their weight change, and also meet every third month for sessions at the OOC. In FBSFT, following assessment, families participate in 17 weekly sessions at the OOC, in which each family works on changing lifestyle behaviors using a structured cognitive-behavioral, socio-ecological approach targeting both parents and children with strategies for behavioral maintenance and sustainable weight change. Outcome variables include body mass index (BMl; $\left.\mathrm{kg} / \mathrm{m}^{2}\right)$, BMI standard deviation score (SDS) and percentage above the IOTF definition of overweight, waist-circumference, body composition (bioelectric impedance (BIA) and dual-X-rayabsorptiometry (DXA)), blood tests, blood pressure, activity/inactivity and sleep pattern (measured by accelerometer), as well as questionnaires measuring depression, general psychological symptomatology, self-esteem, disturbed eating and eating disorder symptoms. Finally, barriers to treatment and parenting styles are measured via questionnaires.
\end{abstract}

Discussion: This is the first systematic application of FBSFT in the treatment of obesity among youth in Norway. The study gives an opportunity to evaluate the effect of FBSFT implemented in routine clinical practice across a range of youth with severe obesity.

Trial registration: ClinicalTrails.gov NCT02687516. Registered 16th of February, 2016

Keywords: Childhood obesity, Randomized controlled trial, Family-based behavioral treatment

\footnotetext{
* Correspondence: Hanna.Flekoy.Skjakodegard@hib.no

'Department of Medicine, the Obesity Outpatient Clinic, Haukeland

University Hospital, N-5021 Bergen, Norway

${ }^{9}$ Department of Physiotherapy, Haukeland University Hospital, Bergen,

Norway

Full list of author information is available at the end of the article
} 


\section{Background}

There has been a global increase in the prevalence and severity of overweight and obesity amongst both adults and children [1, 2]. The World Health Organization (WHO) states that childhood obesity is one of the most serious public health challenges of the 21st century [2].

Childhood obesity is associated with a wide array of negative health consequences such as psychosocial problems, obstructive sleep apnea, increased cardio-metabolic risk and Type 2 Diabetes [3-5], resulting in considerable burden for the individual child as well as socio-economic consequences [6, 7]. In addition, childhood obesity has great impact on adulthood, and is associated with adult obesity [8] and all-cause mortality in adulthood [9]. A recent study [9] with 2.3 million participants between the ages of 16 and 19 years found that a graded increased risk for cardiovascular and all-cause mortality during 40 years of follow-up started among participants with a body mass index (BMI; $\mathrm{kg} / \mathrm{m}^{2}$ ) currently accepted within the normal range (from 50 th to 74 th percentiles).

The need for developing potent treatments for childhood obesity has been recognized for decades and a substantial body of treatment research exists [10-12]. There is also substantial evidence indicating that it is difficult to get more than modest weight loss when treating adolescents with severe obesity $[13,14]$.

Several reviews and meta-analyses have summarized the results of different childhood obesity treatments and provided recommendations as to which treatments are the most efficacious and which treatment components are predictive of favorable treatment outcomes [11, 12, 15, 16]. Specifically, lifestyle interventions that teach behavioral techniques that focus on incorporating the behavior changes into daily life routines, and are delivered in a family-based format (i.e., targeting both the child and parent), seem to be the most efficacious treatment for childhood obesity [11, 15-18]. However, it is important to note that much of this research has been conducted within efficacy trials. Thus, effectiveness trials, which focus on the applicability and validity of the treatment in usual health care settings with a less selected group of participants, are warranted, as few have been conducted [19].

Childhood obesity treatment of medium-to high intensity and longer duration have been found to be most efficacious [20]. Best effect for treatments that included more than 26 sessions are found in one review [20]. There is however some discussion about how prolonged the treatment needs to be to yield meaningful weight loss outcomes. For instance, one review suggests that interventions that last about four months are as efficacious as treatments of longer duration [21]. A meta-analyses of intervention studies from 2002-2015 [18] finds that weight loss treatment for children has evolved over the past decade, especially in that recent studies report longer duration and follow-up than in the 1990s [18]. Despite these findings that indicate the importance of higher-intensity treatment, many national guidelines for addressing childhood obesity recommend weight surveillance and brief lifestyle counseling in primary care service for children with obesity and their families [22]. However, this approach is not effective, at least not for children and adolescents with severe obesity [22, 23].

Recently, there has been consensus that obesity is a disease [24]. Classifying obesity as a chronic health condition increases the understanding of the fact that extended follow-up after an intensive treatment phase is necessary for weight loss maintenance. Long-term maintenance of treatment effects is a challenge with obesity treatment across both adults and children [25-27]. Family-based behavioral treatments for childhood obesity have, importantly, demonstrated promising long-term effects compared to other treatments $[15,28]$. A ten-year follow-up study found that $34 \%$ of the participants who entered the treatment program at age 6-12 had reduced their percent overweight by at least $20 \%$, and $30 \%$ had BMIs that were no longer in the obese range [28].

Dropout is a substantial concern in treatment of both adult and pediatric obesity $[11,29,30]$, but only a limited number of studies have examined the predictors for dropout $[29,31,32]$. Studies show mixed results regarding barriers to treatment participation. Some studies have found higher number of barriers in families who end the treatment program prematurely $[32,33]$, while another found few differences in degree of barriers among treatment completers compared to non-completers [31]. Post-treatment reported barriers in prior studies include high degree of family stressors, parent-adolescent conflict, lack of time and interest, interference with school schedules, disappointment with amount of weight-loss, and treatment taking place too far from home [31, 32, 34]. Further research on predictors for dropout and barriers to treatment participation, both among non-completers and completers, has important implications for clinical practice [30].

Another subject of clinical importance is to identify pretreatment factors related to poorer treatment response. Together with knowledge about predictors for dropout and barriers to treatment participation, these factors can identify at risk groups and tailor childhood obesity treatment to increase treatment response. Several pre-treatment factors have been associated with weight loss outcomes in previous research on childhood obesity treatment [30, 31, 35-38], including child age, gender, child initial weight status $[36,38]$, child mental health problems $[36,37]$, and parents' degree of motivation [30, 31, 35, 36]. However, there seems to be a lack of studies investigating the influence of family variables and broader social network support as predictors of treatment response. 
The aim of this paper is to describe and explain the design and evaluation of the Family-based behavioral treatment of childhood obesity (FABO)-study targeting children and adolescents with obesity and their families. The description of the study protocol follows the checklist of the CONSORT statement for randomized trials [39] (Additional files 1 and 2).

\section{Methods/Design}

\section{Intervention}

Family-based behavioral social facilitation treatment (FBSFT) is founded on the principles of standard familybased behavioral treatment for obesity, which is the most well documented approach for childhood obesity [11].

FBSFT has an intensive treatment phase including weekly family meetings over 17 consecutive weeks with the same health care worker at the Obesity Outpatient Clinic (OOC), Haukeland University Hospital, Norway. All the health care workers on the multidisciplinary treatment team at the OOC are trained in FBSFT prior to treatment delivery. The multidisciplinary team consists of a pediatrician, nutritionist, physiotherapist, nurse and psychologist. Throughout the study period, the treatment team will have weekly meetings to discuss the patients in addition to monthly supervision sessions with the research team in St. Louis, MO, USA and Pittsburgh, PA, USA through video-conferences.

There are session-specific components and goals for each of the 17 sessions (see Table 1 for complete outline). The treatment targets healthy lifestyle changes in both the children and parents in the areas of diet, physical activity, sedentary activity, sleep and social function. The dietary and physical activity guidance used in the study is based on the Traffic Light Diet [40] in which foods and activities are organized into green, yellow and red groups. Green meaning "go," yellow meaning "sometimes" and red meaning "limit." The treatment focuses on implementing the behavior change across all the different settings in the family members' daily lives (i.e., within the home/family environment, peer network environment, community environment).

Through the treatment sessions, the families are taught a set of behavioral and cognitive techniques for promoting healthy behavior change and dealing with mechanisms that maintain unhealthy lifestyle behaviors:

- Self-monitoring. Both the parents and children monitor their eating, weight and activity on a week-to-week basic going through the intensive treatment phase

- Goal setting, planning and reward systems

- Stimulus control

- Emotion regulation strategies

- Reframing negative automatic thoughts

- Communication and interpersonal skills

- Parenting strategies
- Healthy modeling from the parents

- Healthy methods of self-evaluation and self-assertion

After the intensive treatment phase, the families receive monthly follow-up treatment for 18 months through collaboration between specialty and primary care: monthly follow-up sessions with their school nurse (primary care) and follow-up sessions every third month at the OOC. The focus for these sessions is maintenance of healthy habits.

For families assigned to standard treatment (treatment as usual, TAU), the treatment consists of a post-assessment meeting between a health care worker at the OOC and the family, agreeing on behavioral goals for changing lifestyle, a plan for the implementation of new behaviors and goals for weight loss. Each family also participates in monthly counselling session with their local health care nurse to work on implementing these goals, including measuring their weight change, and also meets every third month for sessions at the OOC for assessments, evaluation of progress and revision of goals. TAU is delivered over the course of 12 months.

\section{Study objectives and hypotheses}

The objectives and hypotheses of the current study are:

- To evaluate the effect of FBSFT compared to TAU for severe childhood and adolescent obesity in a common health care setting. The primary outcome is weight status, assessed as BMI, BMI standard deviation scores (SDSs) and percentages above the IOTF cut-off for overweight (\%IOTF-25) [41]. Secondary outcomes are other weightrelated anthropometric measurements (waist circumference (WC) and Waist-to-height ratio (WHtR) and corresponding SDSs), body composition (BIA, DXA), blood test, blood pressure, eating habits, sleep, physical activity as well as psychological well-being and parenting style.

- We hypothesized that FBSFT will be superior to TAU in improving these parameters both during the intensive treatment period and during the follow-up period.

- To identify predictors of treatment success and treatment drop out with a focus on family variables, socioeconomic status, social network and mental health.

- We expect children from families in which both parents participate in treatment to demonstrate better weight loss outcome and have lower dropout rate.

o We expect children of parents living together to demonstrate better treatment effects and have lower dropout rate.

o We expect that parental (self-reported) weight status will influence treatment effects and dropout 
Table 1 Session topics in family-based behavioral facilitation treatment (FBSFT)

\begin{tabular}{|c|c|c|}
\hline Phase & Session & FBSFT Topic \\
\hline \multirow[t]{6}{*}{ 1. Individual and Home Contex } & $1-2$ & Introduction to the treatment; plan for the Traffic Light Diet; personalized treatment plan \\
\hline & 3 & Healthy and regular eating, communicating with the family about lifestyle changes \\
\hline & 4 & Sedentary activity; sleep routines \\
\hline & 5 & Physical activity; lifestyle activity \\
\hline & 6 & Creating a healthy family and home environment; problem solving skills \\
\hline & 7 & Healthy self-instructions; emotions/stress and eating behavior \\
\hline \multirow[t]{4}{*}{ 2. Peer Contex } & 8 & $\begin{array}{l}\text { Peers as a support for healthy behaviors (arranging healthy activities with others); assessment } \\
\text { of social network }\end{array}$ \\
\hline & 9 & High risk situations (parties, holidays and vacations); prompts for eating and physical activity \\
\hline & 10 & A healthy peer environment; communicating with peers about new and healthy habits \\
\hline & 11 & Taking on Teasing \\
\hline \multirow[t]{4}{*}{ 3. Community Contex } & 12 & Physical activity and the assessment of RED food in the environment/neighborhood \\
\hline & 13 & $\begin{array}{l}\text { To be active in your neighborhood; join groups or teams; to elicit support for healthy habits in your } \\
\text { neighborhood/environment }\end{array}$ \\
\hline & 14 & To fight weight stigmatization; influences from the media; to build a positive self-image and body image \\
\hline & 15 & High risk situations (restaurants and fast food); to focus on healthy habits at school and work \\
\hline \multirow[t]{2}{*}{ 4. Cross-contextual } & 16 & To plan for healthy habits; relapse prevention and consolidating skills across different contexts \\
\hline & 17 & Reviewing goals and skills; ending well; planning ahead \\
\hline
\end{tabular}

rate; higher BMI category will predict poorer treatment effects and higher dropout rate.

o We expect that lower socio-economic status and limited social network will predict poorer treatment effects and higher dropout rate.

o We expect poorer treatment effect and higher dropout rate among participants with psychological comorbidities.

- To evaluate experienced barriers to treatment, and how these factors influence children's and parents treatment response. Barriers to treatment are classified into four groups: competing activities/life stressors, relevance of treatment, treatment issues (logistics) and critical events.

- We expect lower degree of competing activities/ life stressors to predict better treatment response and lower dropout rate.

o We expect higher degree of experienced treatment relevance to predict better treatment response and lower dropout rate.

o We expect lower degree of treatment issues and lower number of critical events to predict better treatment response and lower dropout rate.

- To evaluate FBSFT implementation and acceptability for children, parents and health care workers. This is an exploratory study using a brief interview to investigate parents', children's and health care workers' experiences with the treatment.

\section{Trial design}

The FABO-study is a randomized clinical trial (RCT) using a wait-list control design in which all recruited families of children with severe obesity will receive FBSFT at some point. All families go through initial assessments at the Obesity Outpatient Clinic (OOC) at Haukeland University Hospital, and can choose to give informed consent to participate. If informed consent is given, the families are randomized to either FBSFT ( $\operatorname{arm~A)~or~}$ TAU followed by FBSFT one year later (arm B). The overall study design is summarized in Fig. 1. Flow sheet for the FABO-study.

\section{Blinding}

The data manager and statisticians are blinded to group allocation until analyses are conducted. Blinding of participants and/or the treatment team was not possible due to the nature of the study.

\section{Inclusion criteria}

The sample will consist of 120 children and adolescents (aged 6-18 years) referred to the OOC by their general practitioner. Criteria for admission to the clinic is an International Obesity Task Force (IOTF) $\mathrm{BMI} \geq 35$, or $\geq 30$ [41] with obesity related co-morbidity. The child/adolescent participates in the treatment together with her/his family, such that both the child and a least one of the parents agree to actively participate in the treatment. 


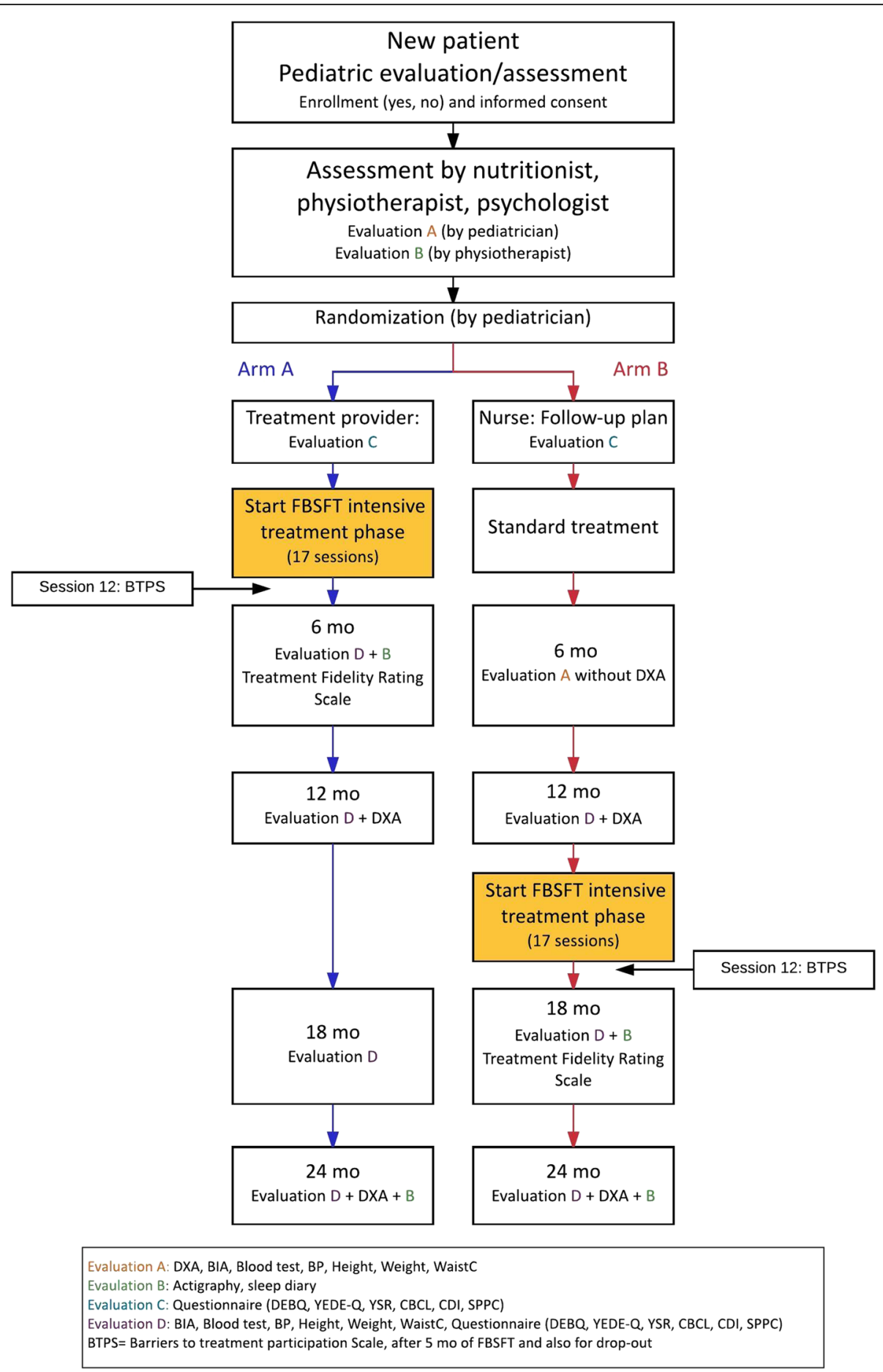

Fig. 1 Flow sheet for the FABO-study

\section{Exclusion criteria}

Families are excluded if either the children or parents experience severe somatic or psychiatric illness that affect weight or adherence to the treatment program, or current participation in other obesity treatment programs.
Strategies to improve adherence to intervention protocol

A standardized checklist for data collection for each child/adolescent participating in the study is created after randomization. Once per month the checklists are reviewed, and participants with missing data are reminded by either text message or phone call to 
complete the assessments. In the intensive treatment phase of FBSFT, sessions are rescheduled so that each family receives the same content, while delivering the sessions within a 6 month period. Sessions are rescheduled within the week when possible, and are combined with the next session or delivered by phone if the family is unable to attend (e.g., due to weather or driving conditions, scheduling conflicts). Health care workers meet weekly for on-site supervision of intervention delivery, and monthly for Skype supervision with the research team in St.Louis, MO, USA and Pittsburgh, PA, USA.

\section{Sample size}

One hundred twenty families will be recruited, with 60 families in each treatment arm. Power estimates were calculated using G*Power, version 3.1.3. [42, 43] With two treatment groups (TAU, FBSFT), 3 measurement points (pre-treatment, 6 months post-treatment and 12 month post-treatment) and a correlation of 0.5 between the measurement points, alpha .05 , power .80 , a small $(f=0.10)$, moderate $(f=0,25)$ and high $(f=0.40)$ effect size would demand respectively sample sizes of 164, 28 and 12 participants to detect a significant group $\mathrm{X}$ time interaction on the primary outcome. For regression analysis of predictors of treatment outcome, a small to moderate effect size is expected $(f=0.15)$, and when testing the increase in $\mathrm{R}^{2}$ by adding 1 predictor to an analysis including 5 predictors in total, a sample size of 55 persons will be needed.

\section{Randomization}

After informed consent is given and the initial assessment is completed, each participant is randomly allocated to either receive FBSFT immediately ( $\operatorname{arm} \mathrm{A}$ ) or following one year of TAU ( $\mathrm{arm} \mathrm{B}$ ). The randomization is performed by the pediatrician and allocation to the two groups is done by extracting a random, sealed envelope from a sealed folder. At the beginning of the study, there were 120 sealed envelopes: 60 with the letter A (for arm A) and 60 with the letter B (for arm B).

\section{Outcome measures}

Measurement points are pre-treatment, post-treatment (i.e., 6 months from pre-treatment), at 12 months, 18 months and 24 months. See Fig. 1 for overview.

\section{Anthropometrical measures}

Trained assessors at the $\mathrm{OOC}$ will measure height and weight for calculation of BMI $(\mathrm{kg} / \mathrm{m} 2)$, WC and body composition (measured with bio impedance (BIA), InBody 720). BMI will be converted to SDS using the extended IOTF $[41,44]$ BMI references. The percentage of the IOTF 25 threshold (\%IOTF-25) is calculated as 100*(BMI/IOTF $25)$, where BMI is the child's weight divided by height squared $\left(\mathrm{kg} / \mathrm{m}^{2}\right)$, and IOTF 25 is the BMI that corresponds to the IOTF threshold for overweight for the child's age and sex. Dual-energy X-ray absorptiometry (DXA)-scans for determining the distribution of fat and muscle tissue will be conducted at the Department of Rheumatology at Haukeland University Hospital.

\section{Physiological measures}

Blood samples will be drawn in the morning after an overnight fast and include measurements of totalcholesterol, high density lipoprotein (HDL), low-density lipoprotein (LDL), triglycerides (TG), aspartate transaminase (ASAT), alanine transaminase (ALAT), creatinine, glycated hemoglobin (HbA1c) and fasting insulin, c-peptide and glucose, thyroid stimulating hormone (THS), free thyroxine (fT4), and C-reactive protein (CRP). A bio-bank for the storing and registering of biological materials has been approved. Trained assessors at the OOC will measure blood pressure.

\section{Physical activity and sleep patterns}

Physical activity and sleep patterns will be measured using Actiwatch 2 (Phillips). The actiwatch devices are wrist-worn accelerometers that records all uni-axial movement over $0.05 \mathrm{G}$ in thirty-second epochs. The actiwatch will be worn on the non-dominant wrist for seven consecutive days. A wrist-worn accelerometer was chosen over hip-worn accelerometers to ensure compliance [45]. Wrist-worn accelerometers are validated for use both as a measure of physical activity, inactivity and sleep and are recommended for use in studies evaluating lifestyle interventions for obesity among children and adults [46-48].

\section{Psychological measures}

The following five questionnaires will be used in the study:

The Child Behavior Checklist (CBCL) [49], a 138-item scale assessing behavioral and emotional symptoms in children that has both a child/youth and parent form. Several studies have provided evidence of the instruments psychometric properties and stability [49-51].

Children's Depression Inventory (CDI) [52], a 27-item self-report measure assessing the cognitive, affective and behavioral symptoms of depression in children (7-17 years). The psychometric properties of the scale have generally been found to be acceptable [53, 54].

Self-Perception Profile for Children (SPPC) [55], a selfreport measure of self-perception or self-esteem in children aged 8 to 14 years widely used for research purposes. The questionnaire includes 36 statements and the children are asked to evaluate to which degree the statement fits their thoughts about themselves. The internal reliability has been demonstrated to be high [56]. 
The Dutch Eating Behavior Questionnaire Child version (DEBQ) [57], a measure of disordered eating behaviors in children and youth. DEBQ consists of three sub-scales: emotional eating, external eating, and restrained eating. The questionnaire is increasingly used for research on youth with overweight and psychometric properties have in general been found acceptable $[58,59]$.

The Youth Eating Disorder Examination-Questionnaire (YEDE-Q) [60], a self-reported measure of eating patterns and eating disorder psychopathology. The YEDE-Q is a self-report version of the Child Eating Disorder Examination (ChEDE) and was designed to include measurements of binge eating in youth [61]. The YEDE-Q has been validated using the ChEDE as an assessment of eating-related pathology in overweight youth [61].

\section{Other measurements}

Barriers to treatment participation scale (BTPS) [62], a 44-item scale developed and validated to address dropout from treatment with out-patient psychological treatment of children and adolescents. The scale is found to yield high levels of internal consistency and to be predictive of treatment dropout and weeks spent in treatment $[62,63]$.

The Parenting Scale (PS) [64], 30-item questionnaire designed to measure different parental disciplines with children and youth. The scale is widely used for research and clinical purposes. The internal consistency and testretest stability have been found to be acceptable to good, and the validity of the instrument has been demonstrated in several studies $[65,66]$.

\section{Planned data analysis}

A two-way MANOVA with one repeated-measure factor (Time: pre-intervention/post-intervention), and one between-group factor (Treatment Group: FBSFT vs. TAU) will be conducted to assess the impact of treatment on primary and secondary outcomes.

A one way within-subjects MANOVA will be used to analyze the effect of time (pre-treatment, post-treatment, $12-, 18-, 24$ months post treatment) on primary and secondary outcomes.

In order to identify factors associated with treatment success and treatment drop out, multiple regression analyses will be conducted. The dependent variables are \%IOTF-25 and drop-out status (yes/no). The predictors are: both parents participating in treatment, parental marital status, self-reported parental weight, socio-economic status and social network, psychological comorbidities.

In order to identify barriers to treatment and how these barriers influence children's and parents' treatment response, multiple regression analyses will be conducted. The dependent variable is \%IOTF-25. The predictors are: competing activities/life stressors, relevance of treatment, treatment issues (logistics) and critical events.

Intention-to-treat analyses will be conducted and effect sizes calculated for treatment effects.

\section{Time plan for the FABO-study}

In this study, we aim to recruit 120 families of children with severe obesity. Enrollment of families to the study began in January 2014 and we anticipate that recruitment will be completed by Autumn 2017.

\section{Discussion}

To our knowledge, this study is the first RCT conducted in Scandinavia evaluating the effect of family-based behavioral treatment of childhood and adolescent obesity delivered in a chronic care model that comprises specialty care and routine clinical practice. The 2011 guidelines from the Norwegian Directorate of Health concerning treatment of overweight children [67], recommend the use of more structured family-based behavioral treatments for severely obese children. However, the availability of such programs has been limited, as well as the possibilities to get training in delivering this type of treatment. This study offers an opportunity to evaluate the effect of this treatment in routine clinical practice. After the study period, FBSFT is likely to be considered as a standard option for treatment, then as a part of stepped care treatment, meaning that non-responders to standard care will advance to this more intensive and targeted treatment.

\section{Additional files}

Additional file 1: SPIRIT 2013 Checklist. Completed checklist for the study protocol article and related document. (DOC $121 \mathrm{~kb}$ )

Additional file 2: Extended information. Study Protocol: A randomized controlled trial evaluating the effect of family-based behavioral treatment of childhood and adolescent obesity - The FABO-study. Description of data: Administrative information requested by SPIRIT 2013 Checklist that are not addressed in the study protocol article. (DOCX $19 \mathrm{~kb}$ )

\section{Abbreviations}

BIA: Bioelectric impedance; BMI: Body mass index; BTPS: Barriers to treatment participation scale; CBCL: The Child Behavior Checklist; CDI: Children's Depression Inventory; ChEDE: Child Eating Disorder Examination; DEBQ: The Dutch Eating Behavior Questionnaire Child version; DXA: Dual-X-rayabsorptiometry; FBSFT: Family-based behavioral social facilitation treatment; IOTF: International Obesity Task Force; OOC: The Obesity Outpatient Clinic; PS: The Parenting Scale; RCT: Randomized controlled trial; SPPC: Self-Perception Profile for Children; TAU: Treatment as usual; WC: Waist circumference; WHO: The World Health Organization; WHtr: Waist-to-height ratio; YEDE-Q: The Youth Eating Disorder Examination-Questionnaire

\section{Acknowledgements}

Vilde Aabel and Ingvild S. Bruserud, Haukeland University Hospital, Bergen, Norway, are members of the treatment team delivering the FBSFT in the FABO-study.

Thanks to Trond Markestad, Haukeland University Hospital, Bergen, Norway, Ståle Pallesen, University of Bergen, Bergen, Norway and Rob Welch, Washington University in St. Louis, St. Louis, Mo, USA for facilitating this project and discussions on project design and content. 


\section{Funding}

The FABO-study is funded by research grants from the Western Norway Regional Health Authority.

\section{Availability of data and materials}

Data collected and used for this study will be part of an electronic regional research register for childhood obesity, "Til normal vekt", situated at Haukeland University Hospital, Norway. The data and materials that will be used for publications from this project can be made available via contacting the study physician.

\section{Authors' contributions}

YSD, RPK, DEW and PBJ designed the FABO-study. PBJ is the research physician. HFS drafted the manuscript. All authors contributed in the writing of the protocol paper and approved the final manuscript.

\section{Competing interests}

All authors declare no conflict of interest.

\section{Consent for publication}

Not applicable.

\section{Ethics approval and consent to participate}

The study has been approved by the Regional Committee for medical and health research ethics (REK) - Western Norway. REK number: 2013/1300. Thorough information about the study and what it will involve will be given to parents and children in written form and in conversation with medical doctor at the OOC, as well as information about the right to withdraw from the study at any point of time. The family will also get information about other treatment options and the possibility to discuss these. Participating parents and children provided written informed consent and assent, respectively.

\section{Author details}

${ }^{1}$ Department of Medicine, the Obesity Outpatient Clinic, Haukeland University Hospital, N-5021 Bergen, Norway. ${ }^{2}$ Department of Clinical Psychology, University of Bergen, Bergen, Norway. ${ }^{3}$ Department of Psychiatry, University of Pittsburgh School of Medicine, Pittsburgh, PA, USA. ${ }^{4}$ Department of Psychiatry, Washington University School of Medicine, St. Louis, MO, USA. ${ }^{5}$ Department of Medicine, Washington University School of Medicine, St. Louis, MO, USA. ${ }^{6}$ Department of Pediatrics, Washington University School of Medicine, St. Louis, MO, USA. ${ }^{7}$ Department of Psychology, Washington University School of Medicine, St. Louis, MO, USA. ${ }^{8}$ Department of Clinical Science, University of Bergen, Bergen, Norway. ${ }^{9}$ Department of Physiotherapy, Haukeland University Hospital, Bergen, Norway

Received: 28 August 2016 Accepted: 8 October 2016

Published online: 21 October 2016

\section{References}

1. Skinner AC, Perrin EM, Skelton JA. Prevalence of obesity and severe obesity in US children, 1999-2014. Obesity (Silver Spring, Md). 2016;24(5):1116-23. doi:10.1002/oby.21497.

2. Final report of the Commission on Ending Childhood Obesity. In: Childhood overweight and obesity. World Health Organization, http://apps.who.int/iris/ bitstream/10665/204176/1/9789241510066_eng.pdf?ua=1 2016. Accessed 10. 082016.

3. Barlow SE. Expert committee recommendations regarding the prevention, assessment, and treatment of child and adolescent overweight and obesity: summary report. Pediatrics. 2007;120 Suppl 4:S164-92. doi:10.1542/peds. 2007-2329C

4. Skinner AC, Perrin EM, Moss LA, Skelton JA. Cardiometabolic risks and severity of obesity in children and young adults. N Engl J Med. 2015;373(14): 1307-17. doi:10.1056/NEJMoa1502821.

5. Kelly AS, Barlow SE, Rao G, Inge TH, Hayman LL, Steinberger J, et al. Severe obesity in children and adolescents: identification, associated health risks, and treatment approaches: a scientific statement from the American Heart Association. Circulation. 2013:128(15):1689-712. doi:10.1161/CIR.0b013e3182a5cfb3.

6. Pelone F, Specchia ML, Veneziano MA, Capizzi S, Bucci S, Mancuso A, et al. Economic impact of childhood obesity on health systems: a systematic review. Obes Rev. 2012;13(5):431-40. doi:10.1111/j.1467-789X.2011.00968.x.
7. von Lengerke T, Krauth C. Economic costs of adult obesity: a review of recent European studies with a focus on subgroup-specific costs. Maturitas. 2011;69(3):220-9. doi:10.1016/j.maturitas.2011.04.005

8. Simmonds M, Llewellyn A, Owen CG, Woolacott N. Predicting adult obesity from childhood obesity: a systematic review and meta-analysis. Obes Rev. 2016;17(2):95-107. doi:10.1111/obr.12334.

9. Twig G, Yaniv G, Levine $H$, Leiba A, Goldberger N, Derazne E, et al. Body-mass index in 2.3 million adolescents and cardiovascular death in adulthood. N Engl J Med. 2016;374:2430-40. doi:10.1056/NEJMoa1503840.

10. Epstein LH, Paluch RA, Roemmich JN, Beecher MD. Family-based obesity treatment, then and now: twenty-five years of pediatric obesity treatment Health Psychol. 2007;26(4):381-91. doi:10.1037/0278-6133.26.4.381.

11. Oude Luttikhuis $H$, Baur L, Jansen $H$, Shrewsbury VA, O'Malley C, Stolk $\mathrm{RP}$ et al. Interventions for treating obesity in children. The Cochrane database of systematic reviews. 2009(1):Cd001872. doi:10.1002/14651858. CD001872.pub2

12. Snethen JA, Broome ME, Treisman P, Castro E, Kelber ST. Effective weight loss for children: a meta-analysis of intervention studies 2002-2015. Worldviews Evid Based Nurs. 2016:13(4):294-302. doi:10.1111/wvn.12156.

13. Knop C, Singer V, Uysal Y, Schaefer A, Wolters B, Reinehr T. Extremely obese children respond better than extremely obese adolescents to lifestyle interventions. Pediatr Obes. 2015;10(1):7-14. doi:10.1111/j.2047-6310.2013.00212.x.

14. Muhlig $Y$, Wabitsch M, Moss A, Hebebrand J. Weight loss in children and adolescents. Dtsch Arztebl Int. 2014;111(48):818-24. doi:10.3238/arztebl.2014.0818.

15. Wilfley DE, Tibbs TL, Van Buren DJ, Reach KP, Walker MS, Epstein LH. Lifestyle interventions in the treatment of childhood overweight: a metaanalytic review of randomized controlled trials. Health Psychol. 2007;26(5): 521-32. doi:10.1037/0278-6133.26.5.521.

16. Altman M, Wilfley DE. Evidence update on the treatment of overweight and obesity in children and adolescents. J Clin Child Adolesc Psychol. 2015;44(4): 521-37. doi:10.1080/15374416.2014.963854.

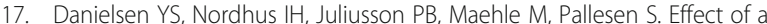
family-based cognitive behavioural intervention on body mass index, selfesteem and symptoms of depression in children with obesity (aged 7-13): a randomised waiting list controlled trial. Obes Res Clin Pract. 2013;7(2): e116-e28. doi:10.1016/j.orcp.2012.06.003.

18. Snethen JA, Broome ME, Treisman P, Castro E, Kelber ST. Effective Weight Loss for Children: A Meta-analysis of Intervention Studies 2002-2015. Worldviews on evidence-based nursing/Sigma Theta Tau International, Honor Society of Nursing. 2016. doi:10.1111/wvn.12156

19. Epstein LH, Wrotniak BH. Future directions for pediatric obesity treatment. Obesity (Silver Spring, Md). 2010;18 Suppl 1:S8-12. doi:10.1038/oby.2009.425.

20. Whitlock EP, O'Connor EA, Williams SB, Beil TL, Lutz KW. Effectiveness of weight management interventions in children: a targeted systematic review for the USPSTF. Pediatrics. 2010;125(2):e396-418. doi:10.1542/peds.2009-1955.

21. Kitzmann KM, Dalton 3rd WT, Stanley CM, Beech BM, Reeves TP, Buscemi J, et al. Lifestyle interventions for youth who are overweight: a meta-analytic review. Health Psychol. 2010:29(1):91-101. doi:10.1037/a0017437.

22. Wake M, Baur LA, Gerner B, Gibbons K, Gold L, Gunn J, et al. Outcomes and costs of primary care surveillance and intervention for overweight or obese children: the LEAP 2 randomised controlled trial. BMJ (Clinical research ed). 2009:339:b3308. doi:10.1136/bmj.b3308.

23. McCallum Z, Wake M, Gerner B, Baur LA, Gibbons K, Gold L, et al. Outcome data from the LEAP (Live, Eat and Play) trial: a randomized controlled trial of a primary care intervention for childhood overweight/mild obesity. Int J Obes. 2007;31(4):630-6. doi:10.1038/sj.ijo.0803509.

24. Farpour-Lambert NJ, Baker JL, Hassapidou M, Holm JC, Nowicka P, O'Malley G, et al. Childhood Obesity Is a Chronic Disease Demanding Specific Health Care-a Position Statement from the Childhood Obesity Task Force (COTF) of the European Association for the Study of Obesity (EASO). Obes Facts. 2015:8(5):342-9. doi:10.1159/000441483.

25. Epstein LH, Myers MD, Raynor HA, Saelens BE. Treatment of pediatric obesity. Pediatrics. 1998:101(3 Pt 2):554-70.

26. Jeffery RW, Drewnowski A, Epstein LH, Stunkard AJ, Wilson GT, Wing RR, et al. Long-term maintenance of weight loss: current status. Health Psychol. 2000;19(1 Suppl):5-16

27. Wadden TA, Butryn ML, Byrne KJ. Efficacy of lifestyle modification for long-term weight control. Obes Res. 2004;12(Suppl):151s-62s. doi:10.1038/oby.2004.282.

28. Epstein $L H$, Valoski A Wing RR, McCurley J. Ten-year outcomes of behavioral family-based treatment for childhood obesity. Health Psychol. 1994;13(5):373-83. 
29. Moroshko I, Brennan L, O'Brien P. Predictors of dropout in weight loss interventions: a systematic review of the literature. Obes Rev. 2011;12(11): 912-34. doi:10.1111/j.1467-789X.2011.00915.x.

30. Gunnarsdottir T, Njardvik U, Olafsdottir AS, Craighead LW, Bjarnason R. The role of parental motivation in family-based treatment for childhood obesity. Obesity (Silver Spring, Md). 2011;19(8):1654-62. doi:10.1038/oby. 2011.59.

31. Braet C, Jeannin R, Mels S, Moens E, Van Winckel M. Ending prematurely a weight loss programme: the impact of child and family characteristics. Clin Psychol Psychother. 2010;17(5):406-17. doi:10.1002/cpp.663.

32. Brennan L, Walkley J, Wilks R. Parent- and adolescent-reported barriers to participation in an adolescent overweight and obesity intervention. Obesity (Silver Spring, Md). 2012;20(6):1319-24. doi:10.1038/oby.2011.358.

33. Inelmen EM, Toffanello ED, Enzi G, Gasparini G, Miotto F, Sergi G, et al. Predictors of drop-out in overweight and obese outpatients. Int J Obes. 2005:29(1):122-8. doi:10.1038/sj.ijo.0802846.

34. Barlow SE, Ohlemeyer CL. Parent reasons for nonreturn to a pediatric weight management program. Clin Pediatr. 2006;45(4):355-60.

35. Braet C. Patient characteristics as predictors of weight loss after an obesity treatment for children. Obesity (Silver Spring, Md). 2006;14(1):148-55. doi:10.1038/oby.2006.18.

36. Moens E, Braet C, Van Winckel M. An 8-year follow-up of treated obese children: children's, process and parental predictors of successful outcome. Behav Res Ther. 2010;48(7):626-33. doi:10.1016/j.brat.2010.03.015.

37. Epstein LH, Wisniewski L, Weng R. Child and parent psychological problems influence child weight control. Obes Res. 1994;2(6):509-15.

38. Goossens L, Braet C, Van Vlierberghe L, Mels S. Weight parameters and pathological eating as predictors of obesity treatment outcome in children and adolescents. Eat Behav. 2009;10(1):71-3. doi:10.1016/j. eatbeh.2008.10.008.

39. CONSORT 2010 checklist of information to include when reporting a randomised trial. Consort Group. 2010. http://www.consort-statement.org/

40. Epstein LH, Wing RR, Koeske R, Valoski A. Effects of diet plus exercise on weight change in parents and children. J Consult Clin Psychol. 1984;52(3):429-37.

41. Cole TJ, Lobstein T. Extended international (IOTF) body mass index cut-offs for thinness, overweight and obesity. Pediatr Obes. 2012;7(4):284-94. doi:10.1111/j.2047-6310.2012.00064.x.

42. Faul F, Erdfelder E, Buchner A, Lang AG. Statistical power analyses using G*Power 3.1: tests for correlation and regression analyses. Behav Res Methods. 2009:41(4):1149-60. doi:10.3758/brm.41.4.1149.

43. Faul F, Erdfelder E, Lang AG, Buchner A. G*Power 3: a flexible statistical power analysis program for the social, behavioral, and biomedical sciences. Behav Res Methods. 2007;39(2):175-91.

44. Juliusson PB, Roelants M, Nordal E, Furevik L, Eide GE, Moster D, et al. Growth references for 0-19 year-old Norwegian children for length/height, weight, body mass index and head circumference. Ann Hum Biol. 2013; 40(3):220-7. doi:10.3109/03014460.2012.759276.

45. Fairclough SJ, Noonan R, Rowlands AV, Van Hees V, Knowles Z, Boddy LM. Wear compliance and activity in children wearing wrist- and Hip-mounted accelerometers. Med Sci Sports Exerc. 2016;48(2):245-53. doi:10.1249/mss. 0000000000000771.

46. Bonomi AG, Westerterp KR. Advances in physical activity monitoring and lifestyle interventions in obesity: a review. Int J Obes. 2012;36(2):167-77. doi:10.1038/ijo.2011.99.

47. Lubans DR, Hesketh K, Cliff DP, Barnett LM, Salmon J, Dollman J, et al. A systematic review of the validity and reliability of sedentary behaviour measures used with children and adolescents. Obes Rev. 2011;12(10): 781-99. doi:10.1111/j.1467-789X.2011.00896.x.

48. Adamo KB, Prince SA, Tricco AC, Connor-Gorber S, Tremblay M. A comparison of indirect versus direct measures for assessing physical activity in the pediatric population: a systematic review. Int J Pediatr Obes. 2009;4(1):2-27. doi:10.1080/ 17477160802315010

49. Achenbach TM. Manual for child behavior checklist/4-18 and 1991 profile. Burlington: University of Vernibtm Department of Psychiatry; 1991.

50. Goodman R, Scott S. Comparing the Strengths and Difficulties Questionnaire and the Child Behavior Checklist: is small beautiful? J Abnorm Child Psychol. 1999;27(1):17-24.

51. Novik TS. Validity of the Child Behaviour Checklist in a Norwegian sample Eur Child Adolesc Psychiatry. 1999;8(4):247-54.

52. Kovacs M. Children's Depression Intenventory (CDI). New York: Multi-Health Systems Inc; 1992.
53. Craighead WE, Curry JF, Ilardi SS. Relationship of Children's Depression Inventory factors to major depression among adolescents. Psychol Assess. 1995;7(2):171-6. doi:10.1037/1040-3590.7.2.171.

54. Craighead WE, Smucker MR, Craighead LW, Ilardi SS. Factor analysis of the Children's Depression Inventory in a community sample. Psychol Assess. 1998;10(2):156-65. doi:10.1037/1040-3590.10.2.156

55. Harter, S. Manual for the Self-Perception Profile for Children: Revision of the Perceived Competence Scale for Children. Denver: University of Denver Press; 1985.

56. Muris P, Meesters C, Fijen P. The Self-Perception Profile for Children: further evidence for its factor structure, reliability, and validity. Personal Individ Differ. 2003;35(8):1791-802. doi:10.1016/S0191-8869(03)00004-7.

57. Vanstrien TFJ, Bergers GPA, Defares PB. The dutch eating behavior questionnaire (DEBQ) for assesment of restrained, emotional, and external eating behavioir. Int $J$ Eat Disord. 1986:5:295-315.

58. Banos RM, Cebolla A, Etchemendy E, Felipe S, Rasal P, Botella C. Validation of the Dutch Eating Behavior Questionnaire for Children (DEBQ-C) for use with Spanish children. Nutr Hosp. 2011;26(4):890-8. doi:10.1590/s0212-16112011000400032.

59. Halvarsson K, Sjödén P-O. Psychometric properties of the dutch eating behaviour questionnaire (DEBQ) among 9-10-year-old Swedish girls. Eur Eat Disord Rev. 1998;6(2):115-25. doi:10.1002/(SICI)1099-0968(199806)6:2<115: AID-ERV222>3.0.CO;2-M.

60. Fairburn CG, Beglin SJ. Assessment of eating disorders: interview or selfreport questionnaire? Int J Eat Disord. 1994:16(4):363-70.

61. Goldschmidt AB, Doyle AC, Wilfley DE. Assessment of binge eating in overweight youth using a questionnaire version of the Child Eating Disorder Examination with Instructions. Int J Eat Disord. 2007;40(5):460-7. doi:10.1002/eat.20387.

62. Kazdin AE, Holland L, Crowley M, Breton S. Barriers to Treatment Participation Scale: evaluation and validation in the context of child outpatient treatment. J Child Psychol Psychiatry. 1997;38(8):1051-62.

63. Colonna-Pydyn C, Gjesfjeld CD, Greeno CG. The factor structure of the barriers to treatment participation scale (BTPS): implications for future barriers scale development. Admin Pol Ment Health. 2007;34(6):563-9. doi:10.1007/s10488-007-0139-6.

64. Arnold DS, O'Leary SG, Wolff LS, Acker MM. The parenting scale: A measure of dysfunctional parenting in discipline situations. Psychol Assess. 1993;5:133-44.

65. Rhoades KA, O'Leary SG. Factor structure and validity of the parenting scale. J Clin Child Adolesc Psychol. 2007;36(2):137-46. doi:10.1080/ 15374410701274157

66. Prinzie $P$, Onghena $P$, Hellinckx W. Reexamining the Parenting Scale: Reliability, factor structure, and concurrent validity of a scale for assessing the discipline practices of mothers and fathers of elementary-school-aged children. Eur J Psychol Assess. 2007:23(1):24-31. doi:10.1027/1015-5759.23.1.24.

67. Nasjonale faglige retninglinjer for primærhelsetjenesten. Forebygging og behandling av overvekt og fedme hos barn og unge. Oslo: The Norwegian Directorate of Health/Helsedirektoratet; 2010.

\section{Submit your next manuscript to BioMed Central and we will help you at every step:}

- We accept pre-submission inquiries

- Our selector tool helps you to find the most relevant journal

- We provide round the clock customer support

- Convenient online submission

- Thorough peer review

- Inclusion in PubMed and all major indexing services

- Maximum visibility for your research

Submit your manuscript at www.biomedcentral.com/submit 